\title{
Riqueza de mudas de espécies florestais nativas potencialmente produzidas na Bacia do Rio Grande, MG
}

Carlos Delano Cardoso de Oliveira ${ }^{1 *}$, Luciana de Moura Gonzaga ${ }^{1}$, Josina Aparecida de Carvalho ${ }^{1}$ Lucas Amaral de Melo Antônio Claudio Davide ${ }^{1}$, Soraya Alvarenga Botelho ${ }^{1}$

${ }^{1}$ Universidade Federal de Lavras, Av. Doutor Sylvio Menicucci, 1001, C P 3037, Kennedy, CEP 37200-000, Lavras, MG, Brasil

*Autor correspondente:

delhano@yahoo.com.br

Termos para indexação:

RENASEM1

Produção de mudas

Restauração ecológica

Index terms:

RENASEM

Seedling production

Ecological restoration

Histórico do artigo:

Recebido em 31/05/2016

Aprovado em 27/05/2017

Publicado em 30/06/2017

doi: 10.4336/2017.pfb.37.90.1342
Resumo - A restauração ecológica tem papel fundamental na retomada da integridade dos ecossistemas, a partir do restabelecimento da riqueza de espécies e estrutura das comunidades vegetais. Assim, caso a decisão seja pela realização de plantio, a produção de mudas florestais com elevada riqueza específica subsidia o sucesso dessa prática. O objetivo deste estudo foi analisar a riqueza de mudas de espécies florestais nativas potencialmente produzidas em viveiros na Bacia do Rio Grande, sul de Minas Gerais. A análise foi baseada no Catálogo das Árvores Nativas de Minas Gerais, considerando as espécies presentes nos subdomínios fitogeográficos Alto Rio Grande, Mantiqueira Sul, Planalto de Poços de Caldas e Noroeste. Para análise das espécies com potencial de produção nos viveiros da Bacia do Rio Grande, foram utilizados os dados do registro nacional de sementes e mudas (RENASEM) de 2013. Foram encontradas 1.251 espécies de ocorrência natural, 102 espécies cadastradas e 49 produtores registrados, distribuídos em 36 municípios. Apenas cinco espécies presentes na lista oficial de espécies ameaçadas de extinção foram cadastradas pelos produtores registrados. A riqueza de espécies florestais cadastradas por produtores registrados no RENASEM na região estudada é muito baixa, o que pode comprometer o sucesso dos projetos de restauração ecológica.

\section{Richness of native forest species seedlings potentially produced in Rio Grande Basin, Minas Gerais State, Brazil}

\begin{abstract}
Ecological restoration plays a fundamental role in ecosystem integrity recovery from restoration of species richness and structure of plant communities. Thus, if the decision is for planting, the production of forest seedlings with high species richness subsidizes the success of this practice. The aim of this study was to analyze the richness of native tree species potentially produced in nurseries in Rio Grande Basin in Southern Minas Gerais State. The analysis was based on the Native Trees Catalogue of Minas Gerais, considering the species present in the phytogeographic subdomains of Upper Rio Grande, South Mantiqueira, Poços de Caldas Plateau and Northwest. For the analysis of species with potential to be grown in nurseries in the Basin, data from the national register of seed and seedlings (RENASEM) from 2013 were used. It was found 1.251 species naturally occurring, 102 species indexed and 49 registered producers, distributed in 36 municipalities. Only five species listed in the official threatened species list were indexed by registered producers. The richness of tree species indexed by registered producers in RENASEM in the studied region is very low, which may compromise the success of ecological restoration projects.
\end{abstract}




\section{Introdução}

A expansão das fronteiras agrícolas, a construção de hidrelétricas e a urbanização são os grandes responsáveis pelo desmatamento da vegetação tropical (Barbosa et al., 2003). No Brasil, os domínios da Mata Atlântica e do Cerrado são os que mais sofrem com a pressão antrópica e, devido ao alto grau de endemismo e devastação, são consideradas áreas prioritárias para conservação da biodiversidade - hotspots (Myers et al., 2000).

A restauração ecológica é uma alternativa para minimizar os danos causados ao ambiente, retomar os serviços ecossistêmicos e conservar a biodiversidade (Chazdon, 2008; Rio de Janeiro, 2010; Rodrigues et al., 2011). Ao visitar a área a ser restaurada e realizar o diagnóstico local, a decisão pode ser pela realização do plantio de mudas, uma técnica de restauração ativa que é a mais utilizada na restauração de florestas (Sampaio et al., 2007). Essa iniciativa é necessária quando um ecossistema é incapaz de retornar naturalmente a sua condição anterior ao distúrbio ou quando esse processo demandaria tempo demasiado (Society for Ecological Restoration, 2004; Rodrigues et al., 2009; Brancalion et al., 2010).

Um dos princípios básicos para restauração ecológica é a tentativa de reconstrução da riqueza de espécies que ocorria naturalmente no ambiente em questão (Rodrigues et al., 2009; Assis et al., 2013) ou é observada em um ecossistema de referência (Society for Ecological Restoration, 2004). Essa riqueza é fundamental para o funcionamento dos ecossistemas e garantia de serviços ecossistêmicos (Isbell et al., 2011). A elevada riqueza de espécies arbóreas propicia um aumento na quantidade de nichos disponíveis, o que leva ao aumento do número de espécies associadas (Larjavaara, 2008). Isso permite a representação de todos os grupos funcionais característicos dos ecossistemas, promovendo seu desenvolvimento e equilíbrio por meio da manutenção da estrutura adequada de comunidade (Society for Ecological Restoration, 2004; Brancalion et al., 2010; Durigan et al., 2010).

De forma geral, a intensificação das iniciativas de restauração ecológica e o aumento das exigências legais têm levado a um crescimento na demanda por mudas de espécies arbóreas nativas da flora brasileira (Santos \& Queiroz, 2011; Silva et al., 2016). Como exemplos, o Cadastro Ambiental Pural (CAR) e o Plano de Regularização Ambiental (PRA) (Brasil, 2012) são instrumentos legais de regularização ambiental dos imóveis rurais que induzirão a recuperação e a restauração de novas áreas, tais como as Áreas de Preservação Permanente (APP) e Reserva Legal (RL) do imóvel rural (Chaves et al., 2015; Instituto de Pesquisa Econômica Aplicada, 2015).

No que diz respeito à produção de mudas de espécies florestais nativas, uma das maiores dificuldades é o alcance de elevada riqueza específica (Rede Mata Atlântica de Sementes Florestais, 2007; Viani \& Rodrigues, 2007; Santos \& Queiroz, 2011; Brancalion et al., 2012). Essa dificuldade ocorre devido a uma série de fatores, como os diferentes comportamentos fisiológicos das espécies, que implicam em diferentes técnicas de manejo, dificuldades de obtenção de sementes, altos índices de predação e falta de conhecimento sobre a silvicultura, fenologia e fisiologia dessas espécies (Viani \& Rodrigues, 2007; Alonso et al., 2014; Silva et al., 2016; Turchetto et al., 2016). Esses fatores tornam grande a discrepância entre o número de espécies que ocorrem naturalmente em ambientes naturais e o número de espécies produzidas em viveiros (Santos \& Queiroz, 2011).

No Brasil, a produção de mudas é regulamentada pela Lei 10.711 de 5 de agosto de 2003 (Brasil, 2003), que instituiu o Sistema Nacional de Sementes e Mudas (SNSM), e Instrução Normativa $\mathrm{n}^{\mathrm{o}} 56$ de 8 de dezembro de 2011 (Brasil, 2011). Essas legislações visam à garantia da qualidade e identidade do material de multiplicação e reprodução vegetal produzido, comercializado e utilizado em todo território nacional. O SNSM compreende, dentre outras atividades, o Registro Nacional de Sementes e Mudas (RENASEM) e o Registro Nacional de Cultivares (RNC).

O RENASEM consiste na identificação de pessoas físicas e jurídicas que exercem atividades de produção, beneficiamento, embalagem, armazenamento, análise, comércio, importação e exportação de sementes e mudas no Brasil. O RNC, por sua vez, é a base do SNSM em que as cultivares devem estar inscritas. Neste contexto, oficialmente possuem direito de produção e comercialização apenas aqueles produtores que possuem cadastro no RENASEM, cujas espécies produzidas estejam registradas no RNC (Brasil, 2003).

Assim, neste estudo objetivou-se avaliar a riqueza de mudas de espécies florestais nativas potencialmente produzidas em viveiros localizados na Bacia do Rio Grande, no sul de Minas Gerais e registrados no RENASEM. 


\section{Material e métodos}

\section{Área do estudo}

A Bacia Hidrográfica do Rio Grande é reconhecida nacionalmente por sua força energética, responsável pela geração de $7.640 \mathrm{MW}$, abrigando treze usinas hidrelétricas. Possui cerca de $145.000 \mathrm{~km}^{2}$ de área de drenagem e sua localização compreende os estados de Minas Gerais e São Paulo (Companhia Energética de Minas Gerais, 2016).

O Rio Grande nasce na Serra da Mantiqueira, em Bocaina de Minas, MG, e percorre $1.306 \mathrm{~km}$ até o Rio Paranaíba, formando o Rio Paraná. Sua abrangência contempla uma grande variedade de ambientes, compreendendo desde os ambientes típicos de Cerrado, até áreas montanhosas típicas da Mata Atlântica (Companhia Energética de Minas Gerais, 2016; Comitê de Bacia Hidrográfica CBH Grande, 2016).

O estudo em questão foi realizado na Bacia do Rio Grande, no sul de Minas Gerais, mais especificamente nas sub-bacias Alto Rio Grande (GD1), Vertentes do Rio Grande (GD2), entorno do Reservatório de Furnas (GD3), Verde (GD4), Sapucaí (GD5), Mogi Guaçu/ Pardo (GD6) e Médio Grande (GD7) (Figura 1). Essas sub-bacias abrangem uma área de aproximadamente 7.577.222 ha e contemplam 196 municípios.

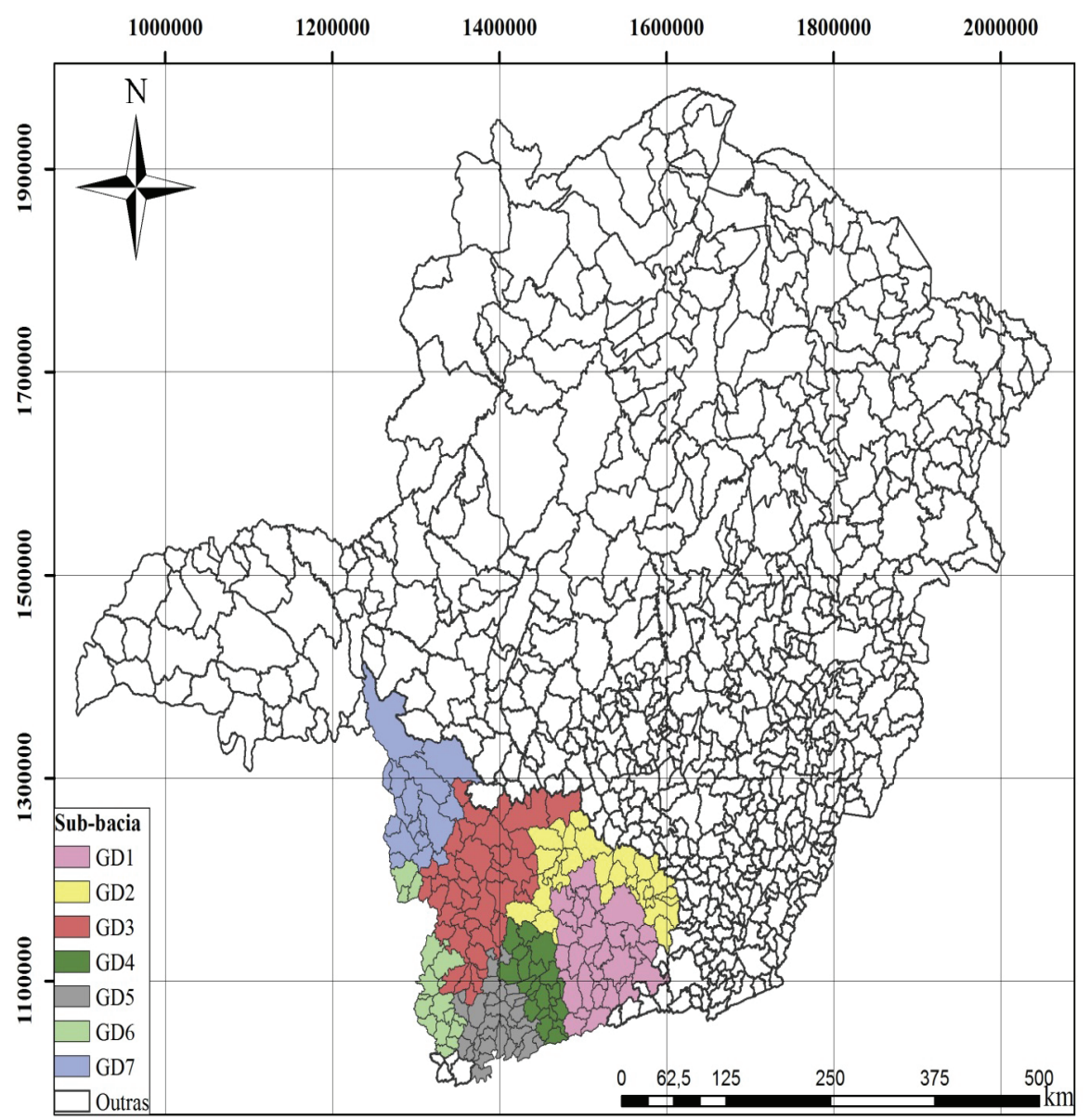

Figura 1. Delimitações das sub-bacias e municípios que compõem a Bacia do Rio Grande, no sul do Estado de Minas Gerais. Onde GD1, GD2, GD3, GD4, GD5, GD6 e GD7 são, respectivamente, as sub-bacias Alto Rio Grande, Vertentes do Rio Grande, entorno do Reservatório de Furnas, Verde, Sapucaí, Mogi Guaçu/ Pardo e Médio Grande. 


\section{Análise da riqueza de espécies florestais nativas}

O levantamento das espécies arbóreas nativas produzidas nos viveiros da região do estudo foi feito com base nos dados do registro nacional de sementes e mudas (RENASEM) de 2013, que foram solicitados à coordenadoria de sementes e mudas do Ministério da Agricultura, Pecuária e Abastecimento (MAPA). Os dados continham informações de todos os produtores cadastrados no Brasil (nome, número de registro no RENASEM, data de validade do registro, $\mathrm{CPF}$ ou CNPJ, atividade - produtor de sementes ou mudas, logradouro e telefone para contato) e das espécies (nome científico e popular) cadastradas por eles. Como não foi possível obter os dados do relatório anual dos viveiros (que contém as espécies e respectivas quantidades produzidas por ano), a lista contemplou as espécies potencialmente produzidas por cada produtor registrado.

A identificação das espécies nativas ocorrentes na região de estudo foi realizada com base em Oliveira Filho (2006). As espécies consideradas neste trabalho foram aquelas que apresentam os hábitos arvoreta, arbóreo e palmeira, pertencentes aos subdomínios fitogeográficos que ocorrem na região sul do Estado de Minas Gerais (ver Oliveira Filho, 2006): Alto Rio Grande, Mantiqueira Sul, Planalto de Poços de Caldas e Noroeste. A atualização dos nomes científicos das espécies foi baseada na Lista de Espécies da Flora do Brasil (Flora do Brasil 2020, 2016).

Inicialmente, foram identificados os municípios pertencentes as sete sub-bacias que formam a Bacia do Rio Grande no sul de Minas Gerais. Esse procedimento foi realizado com base no site oficial do Comitê de Bacia Hidrográfica do Rio Grande (2016). Posteriormente, esses municípios foram usados como critério para filtragem dos dados brutos do RENASEM. Os dados dos produtores de mudas de espécies florestais nativas nos municípios da bacia do Rio Grande cadastrados no RENASEM, o município de localização e as espécies registradas por cada produtor foram organizados em planilha eletrônica.

A planilha de dados das espécies produzidas obtida a partir dos registros no RENASEM foi comparada com a planilha de espécies de ocorrência natural obtida a partir do Catálogo das Árvores Nativas de Minas Gerais (Oliveira Filho, 2006). As espécies constantes nos registros dos produtores de mudas, mas que não foram encontradas na planilha de espécies nativas foram eliminadas da planilha do RENASEM, pois não foram consideradas espécies naturalmente ocorrentes em uma das sete sub-bacias da Bacia do Rio Grande no sul de Minas Gerais. Do mesmo modo, espécies listadas no Catálogo das Árvores Nativas, mas não encontradas entre as espécies da listagem dos produtores registrados no RENASEM foram consideradas espécies nativas não produzidas em uma ou mais das sub-bacias analisadas.

\section{Análise das espécies florestais nativas ameaçadas de extinção}

O levantamento das espécies da flora nativa ameaçadas de extinção foi realizado com base na Portaria ${ }^{\circ} 443$, de 17 de dezembro de 2014 (Brasil, 2014b), disponibilizada pelo Ministério do Meio Ambiente (MMA), que também apresenta o grau de risco de extinção para cada espécie e ressalta as espécies que já constavam na lista elaborada em 2008.

A lista das espécies ameaçadas de extinção do MMA foi comparada com o número total de espécies obtidas no Catálogo das Árvores Nativas de Minas Gerais (Oliveira Filho, 2006), usando o mesmo critério definido para os subdomínios fitogeográficos. Esta análise foi realizada para estimar o número total de espécies que ocorrem na região e que foram classificadas como ameaçadas de extinção. Uma mesma avaliação foi realizada com embasamento na planilha criada por meio da comparação dos dados obtidos na lista do RENASEM e do Catálogo das Árvores Nativas de Minas Gerais. Deste modo, foi possível obter o número de espécies cadastradas pelos viveiros registrados no RENASEM e que ocorrem naturalmente nos subdomínios fitogeográficos definidos. Essa listagem também foi comparada com a lista de espécies ameaçadas de extinção.

\section{Resultados}

De acordo com o Catálogo das Árvores Nativas de Minas Gerais (Oliveira Filho, 2006), 1.251 espécies de hábitos arvoreta, arbóreo e palmeira ocorrem naturalmente nos subdomínios fitogeográficos do Alto Rio Grande, Mantiqueira Sul, Planalto de Poços de Caldas e Noroeste. Essas espécies estão distribuídas por 99 famílias, sendo as famílias Fabaceae, Myrtaceae e Melastomataceae aquelas com maior número de espécies (162, 130 e 78, respectivamente), correspondendo a $29,58 \%$ da riqueza total.

Segundo dados do RENASEM, um único produtor no município de Varginha tem registradas 462 espécies, 
ou seja, $97,47 \%$ de todas as espécies cadastradas na Bacia do Rio Grande, sendo estas distribuídas por 66 famílias. Entretanto, os dados de produção de mudas desse produtor foram retirados da análise, pela grande diferença em relação aos demais, o que poderia gerar resultados distorcidos da realidade.

Assim, a análise dos dados do RENASEM resultou em 49 produtores de mudas de espécies florestais nativas cadastrados em toda Bacia do Rio Grande no sul de Minas Gerais, até fevereiro de 2013. Esses produtores se localizavam em 36 municípios, o que equivale a apenas $16,59 \%$ dos municípios que compõem a Bacia do Rio Grande no sul de Minas Gerais.

O levantamento dos dados do RENASEM mostrou que eram, em média, 5,14 municípios com produtores cadastrados por sub-bacia (Tabela 1). A sub-bacia GD3 (Entorno do Reservatório de Furnas) apresentou o maior número de municípios com produtores cadastrados no RENASEM (nove, ou $25 \%$ do total). Por outro lado, as sub-bacias GD4 (Verde), GD6 (Mogi Guaçu/Pardo) e GD7 (Médio Grande) apresentaram o menor número de municípios com produtores cadastrados: apenas quatro cada uma delas (11,11\% do total de municípios).

No que diz respeito ao número de espécies registradas, a sub-bacia GD2 (Vertentes do Rio Grande) apresentou o maior número (71 ou $69,61 \%$ do total de espécies registradas) e a sub-bacia GD5 (Sapucaí) apresentou 10 espécies registradas, equivalendo ao menor número (Tabela 1).
Tabela 1. Número de municípios com produtores de mudas de espécies florestais cadastrados no RENASEM, número de produtores e número de espécies de ocorrência natural registradas em cada uma das sete sub-bacias analisadas.

\begin{tabular}{lccc}
\hline \multicolumn{1}{c}{ Sub-bacia } & Municípios & Produtores & Espécies \\
\hline GD1 (Alto Rio Grande) & 5 & 9 & 28 \\
GD2 (Vertentes do Rio & 6 & 7 & 71 \\
Grande) & & 16 & 47 \\
GD3 (Entorno do & 9 & 3 & 12 \\
Reservatório de Furnas) & 3 & 6 & 10 \\
GD4 (Verde) & 5 & 4 & 20 \\
GD5 (Sapucaí) & 4 & 4 & 28 \\
GD6 (Mogi Guaçu/Pardo) & 4 & 49 & $102^{(1)}$ \\
GD7 (Médio Grande) & 36 & 7,00 & 30,80 \\
Total & 5,14 & 4,47 & 21,59 \\
Média & 1,95 & & \\
Desvio &
\end{tabular}

(1) Valor correspondente ao total de espécies florestais nativas registradas na Bacia do Rio Grande, no sul de Minas Gerais. (diferentes produtores registraram as mesmas espécies).

Quanto à representatividade da riqueza de espécies que ocorrem naturalmente na Bacia do Rio Grande no sul de Minas Gerais, é possível constatar que todas as sub-bacias apresentaram valores extremamente baixos (Figura 2). A sub-bacia GD2 apresentou maior proporção (5,68\% da riqueza de espécies de ocorrência natural), enquanto a sub-bacia GD5 apresentou menor proporção $(0,80 \%)$.

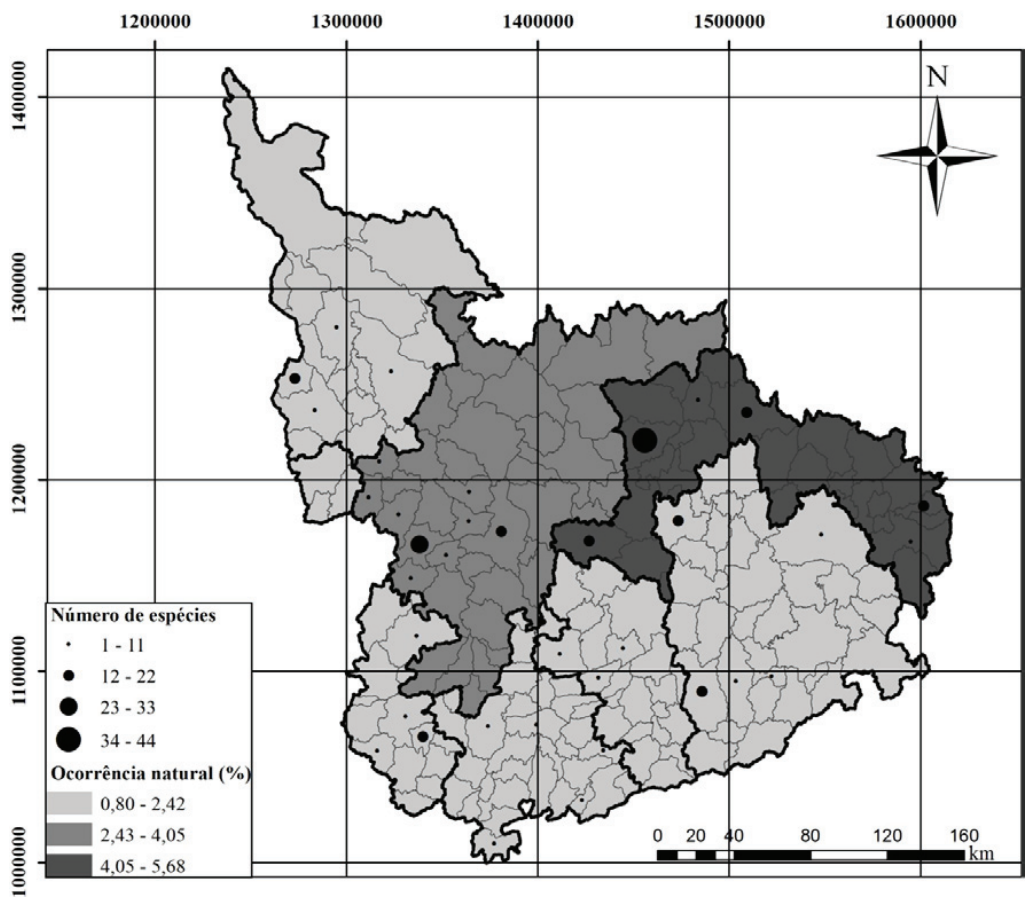

Figura 2. Número de espécies florestais nativas registradas no RENASEM por município, onde existem produtores cadastrados e representatividade da riqueza de espécies registradas nas sub-bacias em relação à riqueza de espécies que ocorrem naturalmente na Bacia do Rio Grande, sul de Minas Gerais. 
Quanto à riqueza de espécies, a média e o desvio padrão nos municípios onde haviam produtores cadastrados foi de 8,36 e 9,63, respectivamente. Os municípios de Campo Belo (sub-bacia GD2), Cabo Verde (sub-bacia GD3) e Ressaquinha (sub-bacia GD2) apresentaram maior riqueza de espécies, apresentando 44, 28 e 22 espécies registradas, respectivamente. Esses valores representaram 43,14\%, 24,51\% e 21,57\% do total de espécies produzidas. Em 24 municípios $(66,66 \%)$ havia menos de dez espécies cadastradas.

Para todos os produtores cadastrados, foram contabilizadas 102 espécies de ocorrência natural vinculadas aos seus registros, sendo estas espécies distribuídas por 33 famílias. Esse número de espécies corresponde a apenas $8,15 \%$ do total de espécies ocorrentes (1.251) na região ocupada pela Bacia do Rio Grande, no sul de Minas Gerais. As famílias Fabaceae, Bignoniaceae e Malvaceae apresentaram a maior riqueza de espécies registradas $(25,8$ e 6 , respectivamente), com $38,24 \%$ do total de espécies cadastradas no Registro.

De todas as 102 espécies cadastradas, Araucaria angustifolia (Bertol.) Kuntze (Araucariaceae), Cedrela fissilis Vell. (Meliaceae), Handroanthus serratifolius (Vahl) S. Grose (Bignoniaceae), Handroanthus roseoalbus (Ridl.) Mattos (Bignoniaceae) e Cedrela odorata L. (Meliaceae) são as espécies mais registradas, sendo provavelmente as mais comumente produzidas na bacia do Rio Grande no sul de Minas, pois constam nos registros de $17,15,15,14$ e 13 produtores, respectivamente.

Das 1.251 espécies que ocorrem naturalmente nos subdomínios fitogeográficos do Alto Rio Grande, Mantiqueira Sul, Planalto de Poços de Caldas e Noroeste (Oliveira Filho, 2006), 29 espécies (Tabela 2) estão presentes na lista oficial de espécies ameaçadas de extinção (Brasil, 2014b). Essas espécies correspondem a 2,31\% do total das espécies de ocorrência natural da Bacia do Rio Grande no Sul de Minas Gerais e estão distribuídas em 15 famílias, sendo destaque as famílias Lauraceae, Fabaceae, Lecythidaceae e Myrtaceae (com 4, 3, 3 e 3 espécies por família, respectivamente).
Tabela 2. Espécies florestais de ocorrência natural da Bacia do Rio Grande, sul de Minas Gerais, que constam na lista oficial de espécies ameaçadas de extinção.

\begin{tabular}{|c|c|c|c|}
\hline $\mathbf{N}^{\circ}$ & Família & Espécie & Categoria \\
\hline 1 & Araucariaceae & $\begin{array}{l}\text { * Araucaria angustifolia } \\
\text { (Bertol.) Kuntze }\end{array}$ & $\mathrm{EN}$ \\
\hline 2 & Arecaceae & Butia capitata (Mart.) Becc. & VU \\
\hline 3 & Arecaceae & * Euterpe edulis Mart. & VU \\
\hline 4 & Bignoniaceae & * Jacaranda rugosa A.H.Gentry & $\mathrm{EN}$ \\
\hline 5 & Bignoniaceae & $\begin{array}{l}\text { Zeyheria tuberculosa (Vell.) } \\
\text { Bureau ex Verl. }\end{array}$ & VU \\
\hline 6 & Cactaceae & $\begin{array}{l}\text { Pilosocereus multicostatus } \\
\text { Ritter }\end{array}$ & $\mathrm{EN}$ \\
\hline 7 & Dichapetalaceae & Stephanopodium engleri Baill. & $\mathrm{EN}$ \\
\hline 8 & Dicksoniaceae & * Dicksonia sellowiana Hook. & EN \\
\hline 9 & Fabaceae & $\begin{array}{l}\text { Apuleia leiocarpa (Vogel) } \\
\text { J.F.Macbr. }\end{array}$ & VU \\
\hline 10 & Fabaceae & $\begin{array}{l}\text { * Dalbergia nigra (Vell.) } \\
\text { Allemão exBenth. }\end{array}$ & VU \\
\hline 11 & Fabaceae & $\begin{array}{l}\text { * Dimorphandra wilsonii } \\
\text { Rizzini }\end{array}$ & $\mathrm{CR}$ \\
\hline 12 & Lauraceae & Aiouea bracteata Kosterm. & VU \\
\hline 13 & Lauraceae & $\begin{array}{l}\text { * Beilschmiedia rigida }(\mathrm{Mez}) \\
\text { Kosterm. }\end{array}$ & $\mathrm{EN}$ \\
\hline 14 & Lauraceae & * Ocotea catharinensis Mez & VU \\
\hline 15 & Lauraceae & $\begin{array}{l}\text { * Ocotea odorifera (Vell.) } \\
\text { Rohwer }\end{array}$ & $\mathrm{EN}$ \\
\hline 16 & Lecythidaceae & $\begin{array}{l}\text { Cariniana legalis (Mart.) } \\
\text { Kuntze }\end{array}$ & $\mathrm{EN}$ \\
\hline 17 & Lecythidaceae & $\begin{array}{l}\text { Couratari pyramidata (Vell.) } \\
\text { Kunth }\end{array}$ & $\mathrm{EN}$ \\
\hline 18 & Lecythidaceae & $\begin{array}{l}\text { Lecythis schwackei (R.Knuth) } \\
\text { S.A.Mori }\end{array}$ & $\mathrm{EN}$ \\
\hline 19 & Meliaceae & Cedrela fissilis Vell. & VU \\
\hline 20 & Meliaceae & Cedrela odorata L. & VU \\
\hline 21 & Myristicaceae & $\begin{array}{l}\text { Virola bicuhyba (Schott ex } \\
\text { Spreng.) Warb. }\end{array}$ & $\mathrm{EN}$ \\
\hline 22 & Myrtaceae & $\begin{array}{l}\text { Myrceugenia bracteosa (DC.) } \\
\text { D.Legrand \&Kausel }\end{array}$ & $\mathrm{EN}$ \\
\hline 23 & Myrtaceae & $\begin{array}{l}\text { Myrceugenia brevipedicellata } \\
\text { (Burret) D.Legrand \&Kausel }\end{array}$ & $\mathrm{EN}$ \\
\hline 24 & Myrtaceae & $\begin{array}{l}\text { Myrcia diaphana (O.Berg) } \\
\text { N.Silveira }\end{array}$ & VU \\
\hline 25 & Primulaceae & Myrsine villosissima A.DC. & $\mathrm{EN}$ \\
\hline 26 & Rubiaceae & $\begin{array}{l}\text { Rudgea jasminoides subsp. } \\
\text { nervosa Zappi \& Anunc. }\end{array}$ & VU \\
\hline 27 & Rubiaceae & $\begin{array}{l}\text { Rudgea sessilis subsp. cipoana } \\
\text { (Standl.)Zappi }\end{array}$ & $\mathrm{EN}$ \\
\hline 28 & Symplocaceae & Symplocos itatiaiae Wawra & EN \\
\hline 29 & Symplocaceae & Symplocos rhamnifolia A.DC. & $\mathrm{EN}$ \\
\hline
\end{tabular}

(*) Espécie que consta na Instrução Normativa 06/2008; CR - criticamente em perigo, EN - em perigo e VU - vulnerável. Fonte: Brasil (2014b). 
Das 29 espécies florestais de ocorrência natural na Bacia do Rio Grande, no sul de Minas Gerais, presentes na lista oficial de espécies ameaçadas de extinção, apenas cinco fazem parte da lista de espécies cadastradas pelos 49 produtores encontrados, a saber: Araucaria angustifolia, Euterpe edulis, Cariniana legalis, Cedrela fissilis e Cedrela odorata.

\section{Discussão}

Os resultados mostram que nos registros do RENASEM no ano de 2013 havia apenas 49 produtores cadastrados em toda a Bacia do Rio Grande, no sul de Minas Gerais, indicando que são poucos os produtores de mudas existentes na Bacia que cumprem o que está previsto na Lei 10.711 de 2003 (Brasil, 2003) e na IN 56 de 2011 (Brasil, 2011).

Em levantamento realizado pela Secretaria de Estado do Ambiente do Estado Rio de Janeiro (Rio de Janeiro, 2010), dos viveiros de espécies florestais nativas existentes no Estado, verificou-se que apenas cinco dos setenta viveiros identificados $(7,14 \%)$ estavam registrados no RENASEM. A falta de um responsável técnico com formação em agronomia ou engenharia florestal, o desconhecimento da legislação vigente e as dificuldades burocráticas no processo de cadastro foram as principais justificativas apresentadas pelos viveiristas para o baixo número de viveiros registrados. No diagnóstico realizado em viveiros no Espírito Santo e Bahia em 2007, a Rede Mata Atlântica de Sementes Florestais (RIOESBA) (2007) contabilizou 19 viveiros $(36,5 \%)$ registrados no RENASEM, dos 52 identificados.

Além do baixo número de viveiros registrados no RENASEM no sul de Minas Gerais, é possível verificar a má distribuição em cada uma das sete sub-bacias (Figura 2). A fim de garantir o suprimento da demanda de mudas e a representatividade de genótipos adaptados às condições locais, é importante a existência de produtores cadastrados no RENASEM de forma bem distribuída em cada sub-bacia. A proximidade de fornecedores ao local onde haverá o plantio diminui o custo de transporte das mudas. Além disso, esses produtores provavelmente fornecem mudas com materiais genéticos mais adaptados às condições locais, o que aumenta suas chances de sucesso no desenvolvimento (Brancalion et al., 2009).

Apesar da alta riqueza de espécies nos domínios fitogeográficos ocorrentes na Bacia do Rio Grande no sul de Minas Gerias (1.251 espécies), é bastante reduzida a riqueza de espécies nativas registradas pelos produtores de mudas cadastrados no RENASEM para essa Bacia (102 espécies). Em avaliação de espécies produzidas em viveiros do Estado do Rio de Janeiro em 2010, Alonso et al. (2014) encontraram 277 espécies nativas da Mata Atlântica. É importante ressaltar que nesse trabalho os autores contabilizaram todas as espécies produzidas, tanto por produtores registrados no RENASEM, quanto por produtores sem registro, mesmo se esses atuavam em desacordo com a legislação brasileira.

Quanto às famílias com maiores números de espécies (Fabaceae, Bignoniaceae e Malvaceae), resultados semelhantes foram obtidos por Alonso et al. (2014) em seu diagnóstico da diversidade de espécies produzidas no Rio de Janeiro. Provavelmente, os resultados semelhantes ocorreram devido às áreas estudadas estarem dentro do domínio da Mata Atlântica e por apresentarem fitofisionomias semelhantes. Além disso, é consenso entre produtores que mudas de muitas espécies destas famílias são mais facilmente produzidas.

O levantamento mostrou que, das duas famílias botânicas com maior riqueza de espécies que ocorrem naturalmente na região analisada (Fabaceae e Myrtaceae) (Carvalho et al., 2007; Fagundes et al., 2007; Souza et al., 2007), a família Fabaceae se destacou quanto ao número de espécies registradas pelos produtores (25). Provavelmente, o menor número de espécies pertencentes à família Myrtaceae que são registradas pelos produtores (5), quando comparado com a riqueza de espécies que ocorrem naturalmente na bacia do Rio Grande no sul de Minas Gerais, está relacionado à recalcitrância de sementes de espécies pertencentes a essa família, o que dificulta seu armazenamento e propagação, característica relatada por CangahualaInocente et al. (2007) e Delgado \& Barbedo (2012).

Dentre as cinco espécies mais cadastradas pelos produtores, $H$. serratifolia e $H$. roseo-alba são amplamente demandadas na arborização urbana. Não obstante, A. angustifolia, C. fissilis e C. odorata são espécies muitas vezes procuradas devido ao interesse econômico-cultural associado à qualidade de suas madeiras. Somados a outros fatores, como a facilidade de produção de mudas de tais espécies, essas características possivelmente sejam as causas da maior motivação para o seu registro por parte de um grande número de produtores.

Corroborando com o resultado da presente pesquisa, Gonçalves et al. (2004), em diagnóstico dos viveiros 
municipais de Minas Gerais, observaram que a maioria dos municípios que possuíam viveiros, produziam mudas para a arborização urbana e para recuperação ambiental, sendo destinadas principalmente à doação. Santos \& Queiroz (2011), ao estudarem viveiros florestais do Sudeste Goiano, também observaram que as mudas de espécies florestais nativas se destinavam, de um modo geral, para paisagismo e alguns projetos de reflorestamento.

Os resultados da pesquisa mostram que apenas cinco espécies que constam na lista de espécies ameaçadas de extinção (Brasil, 2014b) são cadastradas por produtores. A. angustifolia e E. edulis estão listadas como ameaçadas desde o levantamento realizado em 2008 (Brasil, 2008).

Devido à drástica redução de seu tamanho populacional em paisagens antrópicas, as espécies ameaçadas possuem chances mínimas de ocupar áreas restauradas, caso não sejam diretamente inseridas nesses locais, especialmente em plantios. As práticas de restauração ecológica assumem, então, um papel fundamental para reintrodução e manutenção das populações dessas espécies, principalmente pela criação de hábitat para seu restabelecimento, reinserção de suas populações na paisagem e auxílio na conservação e reconexão de fragmentos onde essas espécies possam ocorrer (Brancalion et al., 2010). Assim, é de extrema importância a produção de mudas de espécies nativas ameaçadas de extinção para sua utilização em projetos de restauração ecológica.

Em seu diagnóstico sobre a produção de mudas de espécies nativas no Brasil, o Instituto de Pesquisa Econômica Aplicada (2015) constatou o menor número de viveiros ativos em Minas Gerais, quando comparado com os demais estados da região Sudeste (São Paulo, Rio de Janeiro e Espírito Santo). Minas Gerais, juntamente com o Rio de Janeiro, também apresentou a menor capacidade anual média de produção de mudas de espécies florestais nativas em comparação com os estados de São Paulo e Espírito Santo. Esses resultados mostram que, apesar de estar localizado na região Sudeste do Brasil onde existe uma cadeia de viveiros bem estruturada (Silva et al., 2016) e ser o maior estado da região em área territorial, Minas Gerais possui menor expressão na produção de mudas de espécies nativas.

No Estado de Minas Gerais, a Lei 20.922 de 16 de outubro de 2013 (Minas Gerais, 2013) estabelece normas de apoio e incentivos fiscais para a pessoa física ou jurídica que preservar e conservar a vegetação nativa ou recuperar áreas degradadas com espécies nativas. Um exemplo desse apoio é o fornecimento gratuito de mudas de espécies nativas e insumos por parte do Instituto Estadual de Florestas (IEF) para os produtores rurais que têm como objetivo a recuperação ambiental ou produção florestal (Instituto Estadual de Florestas, 2016).

Se por um lado essa ação do Governo de Minas Gerais estimula as iniciativas de recuperação de áreas degradadas e restauração ecológica por meio da facilitação do acesso a insumos necessários a essas práticas, por outro lado isso pode conter o mercado de produção de mudas. Com o fornecimento gratuito de mudas de espécies nativas, a produção por parte da iniciativa privada pode ficar comprometida naquelas regiões onde existem viveiros do IEF ou parcerias.

Apesar da instituição do Cadastro Ambiental Rural (CAR) e do Programa de Regularização Ambiental (PRA), a Lei $n^{\circ} 12.651$ (Brasil, 2012) não determina características estruturais, funcionais e níveis de composição de espécies a serem utilizados em projetos de restauração ecológica. Isso pode ser justificado pela complexidade de diferenças florísticas e estruturais entre ecossistemas existentes no Brasil, o que incita a necessidade de legislações específicas, em nível estadual ou mesmo por domínio fitogeográfico (Aronson et al., 2011; Chaves et al., 2015).

A ausência de uma legislação específica para recuperação de áreas degradadas e restauração ecológica pode ser a principal justificativa da atual situação de produção de mudas em Minas Gerais (Instituto de Pesquisa Econômica Aplicada, 2015). A falta de especificações legais quanto ao número mínimo de espécies e grupos ecológicos para plantio por unidade de área para o estado, respeitando-se as diferentes realidades e fitofisionomias ocorrentes, se constitui em um entrave à consolidação da riqueza de espécies produzidas em viveiros para a recomposição florestal (Rio de Janeiro, 2010). No Espírito Santo e Bahia foi encontrada uma diversidade média de 26 espécies nativas por viveiro, o que representou um número muito baixo, que também foi justificado por não haver legislação específica para esses estados (Rede Mata Atlântica de Sementes Florestais, 2007).

Em São Paulo, a Resolução SMA n. ${ }^{\circ} 32$ de 3 de abril de 2014 (São Paulo, 2014) tem como objetivo definir procedimentos para aprimorar a restauração no estado (Aronson et al., 2011). Essa Resolução, que serviu como exemplo para outros estados (Durigan et 
al., 2010), sugere um mínimo de 80 espécies florestais nativas de ocorrência regional por projeto de restauração ecológica em áreas de ocorrência das formações de Floresta Ombrófila, Estacional Semidecidual e Savana Florestada. Além disso, ela determina a utilização de pelo menos $5 \%$ de espécies nativas da vegetação regional enquadradas em uma das categorias de ameaça: vulnerável, em perigo, criticamente em perigo ou presumivelmente extinta. De acordo com Brancalion et al. (2010) e Assis et al. (2013), impactos positivos da existência de resoluções como essa no estado de São Paulo são notados na riqueza e número de mudas de espécies nativas produzidas a partir de 2001, após a publicação da primeira versão.

Os benefícios criados pela existência de uma legislação específica, como ocorre em São Paulo (Barbosa et al., 2012), vão além da riqueza de espécies produzidas. A existência de parâmetros que orientem as práticas de restauração ecológica intensificam as ações e tem impacto em toda a cadeia, gerando trabalho, renda, bens e serviços relacionados a essa prática, além dos serviços ambientais prestados pelos ecossistemas restaurados (Brancalion et al., 2010; BenDor et al., 2015; Instituto de Pesquisa Econômica Aplicada, 2015; Silva et al., 2016). Somados à existência de uma legislação com diretrizes aplicáveis, os incentivos fiscais governamentais também são essenciais para a intensificação das práticas de restauração ecológica (Aronson et al., 2011). Em países como África do Sul, Japão, China e Estados Unidos, o setor público fornece apoio financeiro para a restauração de ecossistemas (Durigan et al., 2010).

Embora existam as bases nacionais que compõem o Sistema Nacional de Sementes e Mudas, muitos produtores de mudas de espécies nativas não estão vinculados a esses registros, o que faz com que as informações disponíveis sejam limitadas (Instituto de Pesquisa Econômica Aplicada, 2015). Para inversão dessa situação, é imprescindível o estímulo ao cadastro dos produtores. A maior divulgação do Sistema Nacional de Sementes e Mudas, a diminuição da burocracia envolvida para o cadastro, a facilitação de acesso à informação e o incentivo ao cadastro por meio da redução de impostos, certificação dos produtores cadastrados e apoio técnico e financeiro, são ações com potencial para regularização dos produtores (Rio de Janeiro, 2010; Silva et al., 2016).

A organização de redes de sementes e mudas também tem potencial para aprimorar a riqueza e diversidade nos viveiros. Seu funcionamento se baseia no intercâmbio de espécies e materiais genéticos entre os viveiros que compõem a rede. Essa alternativa é capaz de contornar falhas na frutificação e problemas de coleta, de forma a não comprometer a produção no viveiro, além de contribuir com aumento da riqueza florística e diversidade genética (Brancalion et al., 2009). Por se tratarem de áreas com características climáticas semelhantes, as sub-bacias poderiam ser usadas como unidades para o estabelecimento das redes. O intercâmbio de materiais entre produtores dessas unidades contribuiria significativamente para o desenvolvimento da produção de espécies nativas desses locais.

A organização de uma rede de sementes de espécies florestais nativas da Mata Atlântica e do Cerrado fundamentada pelo envolvimento de produtores, instituições, universidade, etc., com a criação e manutenção de bancos de sementes para as demandas regionais, pode representar uma forma de mitigar a dificuldade de aquisição de sementes.

A combinação de estratégias para obtenção de estoques de plantas é uma forma efetiva de aumentar a riqueza de espécies, a diversidade genética das mudas produzidas e a representatividade dos diferentes grupos funcionais em viveiros de espécies florestais tropicais. Diferentes formas de coletas de sementes e o resgate de plântulas são alternativas que podem contribuir para a restauração de florestas altamente diversas e gerar, também, benefícios socioeconômicos (Calegari et al., 2011; Brancalion et al., 2012; Turchetto et al., 2016).

Vários estudos têm analisado e identificado bons resultados na produção de mudas por meio do resgate de indivíduos naturalmente regenerantes (Viani \& Rodrigues, 2007; Calegari et al., 2011; Turchetto et al., 2016). Dentre as principais vantagens associadas à essa técnica, pode-se citar a adaptação das mudas às condições bioclimáticas regionais, obtenção de mudas com diversidade genética pré-existente e a eliminação de alguns passos da produção de mudas (seleção de matrizes, coleta e beneficiamento de sementes), que aumentam os custos e dificultam o processo (Rodrigues et al., 2009; Calegari et al., 2011; Turchetto et al., 2016). Apesar dessas vantagens, o uso dessa técnica só é permitido como medida mitigadora em áreas cuja vegetação foi suprimida mediante o licenciamento ambiental (Calegari et al., 2011).

A carência de uma infraestrutura adequada de sementes e mudas dificulta a restauração ecológica. Dessa forma, estudos referentes à diversidade de 
mudas produzidas em viveiros são relevantes, pois realizam um diagnóstico da produção, com provável impacto nos reflorestamentos, e subsidiam iniciativas como o Plano Nacional de Recuperação da Vegetação Nativa (PLANAVEG), que possui como meta inicial a restauração de 12,5 milhões de ha nos próximos 20 anos (Brasil, 2014a).

A caracterização do cenário atual por meio de diagnóstico permite aos produtores a identificação das carências da atividade e melhoria na produção, principalmente no que diz respeito à riqueza de espécies (Santos \& Queiroz, 2011). Isso pode ser observado nesse estudo, onde uma área de grande representatividade econômica e socioambiental tem uma oferta de mudas de espécies nativas nos viveiros inferior a $10 \%$ da riqueza de espécies que ocorrem naturalmente na região.

\section{Conclusão}

A riqueza de mudas de espécies registradas pelos produtores cadastrados é baixa e representa menos de $10 \%$ do que ocorre naturalmente na região do estudo. Além disso, poucas espécies que ocorrem naturalmente na Bacia do Rio Grande (Minas Gerais) e que constam na lista oficial de espécies ameaçadas de extinção são cadastradas pelos produtores.

$\mathrm{O}$ número de produtores de mudas cadastrados no RENASEM é baixo e eles estão mal distribuídos na Bacia do Rio Grande, no sul de Minas Gerais. A criação de uma legislação específica em Minas Gerais, levando em consideração as diferentes formações vegetais existentes no estado, é a principal alternativa para alterar essa realidade e impulsionar as ações de restauração ecológica. Além da retomada dos serviços ambientais, a geração de renda, bens e serviços são benefícios gerados com a existência de uma legislação aplicável.

\section{Agradecimentos}

Os autores do estudo agradecem ao Ministério da Agricultura, Pecuária e Abastecimento (MAPA) pelo fornecimento dos dados, a Coordenação de Aperfeiçoamento de Pessoal de Nível Superior (CAPES) pela concessão de bolsas de estudo de pós-graduação, à Fundação de Desenvolvimento Científico e Cultural (FUNDECC) e à Companhia Energética de Minas Gerais (CEMIG) pelo apoio a projetos científicos.

\section{Referências}

Alonso, J. M. et al. Avaliação da diversidade de espécies nativas produzidas nos viveiros florestais do estado do Rio de Janeiro. Floresta, v. 44, n. 3, p. 369-380, 2014.

Aronson, J. et al. What role should government regulation play in ecological restoration? Ongoing debate in São Paulo State, Brazil. Restoration Ecology, v. 19, n. 6, p. 690-695, 2011. DOI: 10.1111/j.1526-100X.2011.00815.x.

Assis, G. B. et al. Uso de espécies nativas e exóticas na restauração de matas ciliares no estado de São Paulo (1957 - 2008). Revista Árvore, v. 37, n. 4, p. 599-609, 2013.

Barbosa, L. M. et al. Práticas e políticas públicas para restauração ecológica a partir de reflorestamentos com alta diversidade de espécies regionais. In: Martins, S. V. (Ed.). Restauração ecológica de ecossistemas degradados. Viçosa, MG: Ed da UFV, 2012. p. 240-261.

Barbosa, L. M. et al. Recuperação florestal com espécies nativas no estado de São Paulo: pesquisas apontam mudanças necessárias. Florestar Estatístico, v. 6, n. 14, p. 28-34, 2003.

BenDor, T. et al. Estimating the size and impact of the ecological restoration economy. Plos One, v. 10, n. 6, p. 1-15, 2015. DOI: 10.1371/journal.pone.0128339.

Brancalion, P. H. S. et al. Improving planting stocks for the Brazilian Atlantic Forest restoration through community-based seed harvesting strategies. Restoration Ecology, v. 20, n. 6, p. 704-711, 2012. DOI: 10.1111/j.1526-100X.2011.00839.x.

Brancalion, P. H. S. et al. Instrumentos legais podem contribuir para a restauração de florestas tropicais biodiversas. Revista Árvore, v. 34, n. 3, p. 455-470, 2010. DOI: 10.1590/S0100-67622010000300010.

Brancalion, P. H. S. et al. Incorporação do conceito da diversidade genética na restauração ecológica. In: Rodrigues, R. R. et al. (Org.) Pacto pela restauração da Mata Atlântica: referencial dos conceitos e ações de restauração florestal. São Paulo: LERF/ESALQ, Instituto BioAtlântica, 2009. p. 37-54.

Brasil. Lei n ${ }^{\circ} 12.651$ de 25 de maio de 2012. Dispõe sobre a proteção da vegetação nativa; altera as Leis nํㅗ 6.938 , de 31 de agosto de 1981 , 9.393, de 19 de dezembro de 1996, e 11.428 , de 22 de dezembro de 2006; revoga as Leis $\mathrm{n} \stackrel{\mathrm{os}}{ }$ 4.771, de 15 de setembro de 1965, e 7.754, de 14 de abril de 1989, e a Medida Provisória n-2.166-67, de 24 de agosto de 2001; e dá outras providências. Diário Oficial [da] República Federativa do Brasil, Brasília, DF, n. 102, p. 1-11, 28 mai. 2012.

Brasil. Ministério da Agricultura, Pecuária e Abastecimento. Instrução Normativa $n^{\circ} 56$, de 8 de dezembro de 2011. Diário Oficial [da] República Federativa do Brasil, Brasília, DF, n. 236, p. 34-41, 9 dez. 2011.

Brasil. Ministério da Agricultura, Pecuária e Abastecimento. Lei n ${ }^{\circ}$ 10.711 , de 3 de agosto de 2003. Dispõe sobre o Sistema Nacional de Sementes e Mudas e dá outras providências. Diário Oficial [da] República Federativa do Brasil, Brasília, DF, n. 150, p. 1-4, 5 ago. 2003. 
Brasil. Ministério do Meio Ambiente. Instrução Normativa nº .06 , de 23 de Setembro de 2008. Lista Oficial da flora brasileira ameaçada de extinção. Diário Oficial [da] República Federativa do Brasil, Brasília, DF, n. 185, p. 75-83, 24 set. 2008.

Brasil. Ministério do Meio Ambiente. PLANAVEG: versão preliminar. Brasília, 2014a. Disponível em: <http://www.mma. gov.br/images/arquivo/80049/Planaveg/PLANAVEG_20-11-14. pdf>. Acesso em: 24 mar. 2017.

Brasil. Ministério do Meio Ambiente. Portaria n ${ }^{\circ} 443$, de 17 de dezembro de 2014. Diário Oficial [da] República Federativa do Brasil, Brasília, DF, Seção 1, p. 110-121, 18 dez. 2014 b.

Calegari, L. et al. Produção de mudas de espécies arbóreas nativas em viveiro via resgate de plantas jovens. Revista Árvore, v. 35, n. 1, p. 41-50, 2011.

Cangahuala-Inocente, G. C. et al. Improvements in somatic embryogenesis protocol in Feijoa (Acca sellowiana (Berg) Burret): Induction, conversion and synthetic seeds. Scientia Horticulturae, v. 111, n. 3, p. 228-234, 2007. DOI: 10.1016/j.scienta.2006.10.030.

Carvalho, W. A. C. et al. Variação espacial da estrutura da comunidade arbórea de um fragmento de floresta semidecídua em Piedade do Rio Grande, MG, Brasil. Revista Brasileira de Botânica, v. 30, n. 2, p. 315-335, 2007. DOI: 10.1590/S0100-84042007000200015.

Chaves, R. B. et al. On the need of legal frameworks for assessing restoration projects success: new perspectives from São Paulo state (Brazil). Restoration Ecology, v. 23, n. 6, p. 754-759, 2015. DOI: 10.1111/rec.12267.

Chazdon, R. L. Beyond deforestation: restoring forests and ecosystem services on degraded lands. Science, v. 320, n. 5882, p. 1458-1460, 2008. DOI: $10.1126 /$ science. 1155365 .

Companhia Energética de Minas Gerais. Bacia do Rio Grande. Disponível em: <http://www.cemig.com.br/pt-br/A_Cemig_e_o_ Futuro/sustentabilidade/nossos_programas/ambientais/peixe_vivo/ Paginas/rio_grande.aspx>. Acesso em: 30 ago. 2016.

Comitê de Bacia Hidrográfica $\mathrm{CBH}$ Grande. A bacia. Disponível em: <http://www.grande.cbh.gov.br/Bacia.aspx>. Acesso em: 30 ago. 2016.

Delgado, L. F. \& Barbedo, C. J. Water potential and viability of seeds of Eugenia (Myrtaceae), a tropical tree species, based upon different levels of drying. Brazilian Archives of Biology and Technology, v. 55, n. 4, p. 583-590, 2012. DOI: 10.1590/S151689132012000400014.

Durigan, G. et al. Normas jurídicas para a restauração ecológica: uma barreira a mais a dificultar o êxito das iniciativas? Revista Árvore, v. 34, n. 3, p. 471-485, 2010. DOI: 10.1590/S010067622010000300011.

Fagundes, L. M. et al. Florística e estrutura do estrato arbóreo de dois fragmentos de florestas decíduas às margens do Rio Grande, em Alpinópolis e Passos, MG, Brasil. Acta Botânica Brasileira, v. 21, n. 1, p. 65-78, 2007. DOI: 10.1590/S0102-33062007000100007.

Flora do Brasil 2020. Rio de Janeiro: Jardim Botânico do Rio de Janeiro, [2016]. Disponível em: <http://floradobrasil.jbrj.gov.br/>. Acesso em: 10 ago. 2016.
Gonçalves, E. O. et al. Diagnóstico dos viveiros municipais no Estado de Minas Gerais. Ciência Florestal, v. 14, n. 2, p. 1-12, 2004. DOI: 10.5902/198050981801.

Instituto Estadual de Florestas. Produção de mudas. Disponível em: $<$ http://www.ief.mg.gov.br/servicos-ief/1616-producao-de-mudas $>$. Acesso em: 30 ago. 2016.

Instituto de Pesquisa Econômica Aplicada. Diagnóstico da produção de mudas florestais nativas no Brasil: relatório de pesquisa. Brasília, DF, 2015. 58 p. Disponível em: <http://www.lerf.eco.br/ img/publicacoes/150507_relatorio_diagnostico_producao.pdf $>$. Acesso em: 14 ago. 2016.

Isbell, F. et al. High plant diversity is needed to maintain ecosystem services. Nature, v. 477, n. 7363, p. 199-202, 2011. DOI: 10.1038/ nature10282.

Larjavaara, M. A review on benefits and disadvantages of tree diversity. The Open Forest Science Journal, v. 1, p. 24-26, 2008. DOI: $10.2174 / 1874398600801010024$.

Minas Gerais. Lei n ${ }^{\circ} 20.922$, de 16 de outubro de 2013. Dispõe sobre as políticas florestal e de proteção à biodiversidade no Estado. Diário Oficial do Estado de Minas Gerais, Belo Horizonte, MG, Seção do Executivo, p. 1-8, 17 out. 2013.

Myers, N. et al. Biodiversity hotspots for conservation priorities. Nature, v. 403, n. 6772, p. 853-858, 2000. DOI: 10.1038/35002501.

Oliveira Filho, A. T. Catálogo das árvores nativas de Minas Gerais: mapeamento e inventário da flora nativa e dos reflorestamentos de Minas Gerais. Lavras: Ed da UFLA, 2006. 423 p.

Rede Mata Atlântica de Sementes Florestais. Diagnóstico dos viveiros florestais de espécies nativas da Mata Atlântica da Bahia e Espírito Santo. Seropédica, 2007. 30 p. Disponível em: <http:// www.if.ufrrj.br/rioesba/pdf/diagnostico_setor_ES_BA.pdf $>$. Acesso em: 14 ago. 2016.

Rio de Janeiro. Secretaria de Estado do Ambiente. Superintendência de Biodiversidade. Diagnóstico da produção de mudas de espécies nativas no estado do Rio de Janeiro. Rio de Janeiro, 2010. 63 p. Disponível em: <http://www.itpa.org.br/wp-content/uploads/ diagnostico_mudas_VF.pdf >. Acesso em: 14 ago. 2016.

Rodrigues, R. R. et al. Large-scale ecological restoration of high-diversity tropical forests in SE Brazil. Forest Ecology and Management, v. 261, n. 10, p. 1605-1613, 2011. DOI: 10.1016/j. foreco.2010.07.005.

Rodrigues, R. R. et al. On the restoration of high diversity forests: 30 years of experience in the Brazilian Atlantic Forest. Biological Conservation, v. 142 , n. 6 , p. 1242-1251, 2009. DOI: 10.1016/j. biocon.2008.12.008.

Sampaio, A. B. et al. Does restoration enhance regeneration of seasonal deciduous forests in pastures in central Brazil? Restoration Ecology, v. 15, n. 3, p. 462-471, 2007. DOI: 10.1111/j.1526100X.2007.00242.x.

Santos, J. J. \& Queiroz, S. E. E. Diversidade de espécies nativas arbóreas produzidas em viveiros. Enciclopédia Biosfera, v. 7, n. 12, p. 1-8, 2011. 
São Paulo. Resolução SMAn ${ }^{\circ}$ 32, de 03 de maio de 2014. Estabelece as orientações, diretrizes e critérios sobre a restauração ecológica no estado de São Paulo e dá providências correlatas. Diário Oficial do Estado, São Paulo, Seção I, p. 36-37, 5 abr. 2014.

Silva, A. P. M. et al. Can current tree seedling production and infrastructure meet an increasing forest restoration demand in Brazil? Restoration Ecology, v.1, 2016. DOI: 10.1111/rec.12470.

Society for Ecological Restoration. Science \& Policy Working Group. SER international primer for ecological restoration. Tucson, 2004. Disponível em: <http:/c.ymcdn.com/sites/www.ser.org/resource/ resmgr/custompages/publications/ser_publications/ser_primer.pdf >. Acesso em: 05 ago. 2016.
Souza, F. N. et al. Composição florística e estrutura de dois fragmentos de Floresta Estacional Semidecidual na Bacia do Rio Grande, Minas Gerais. Revista Brasileira de Biociências, v. 5, n. 52, p. 183-185, 2007.

Turchetto, F. et al. Can transplantation of forest seedlings be a strategy to enrich seedling production in plant nurseries? Forest Ecology and Management, v. 375, p. 96-104, 2016. DOI: 10.1016/j. foreco.2016.05.029.

Viani, R. A. G. \& Rodrigues, R. R. Sobrevivência em viveiro de mudas de espécies nativas retiradas da regeneração natural de remanescente florestal. Pesquisa Agropecuária Brasileira, v. 42, n. 8, p. 1067-1075, 2007. DOI: 10.1590/S0100-204X2007000800002. 